Document downloaded from:

http://hdl.handle.net/10251/159524

This paper must be cited as:

Guardiola, C.; Pla Moreno, B.; Bares-Moreno, P.; Peyton Jones, J. (2019). Integration of intermittent measurement from in-cylinder pressure resonance in a multi-sensor mass flow estimator. Mechanical Systems and Signal Processing. 131:152-165.

https://doi.org/10.1016/j.ymssp.2019.05.052

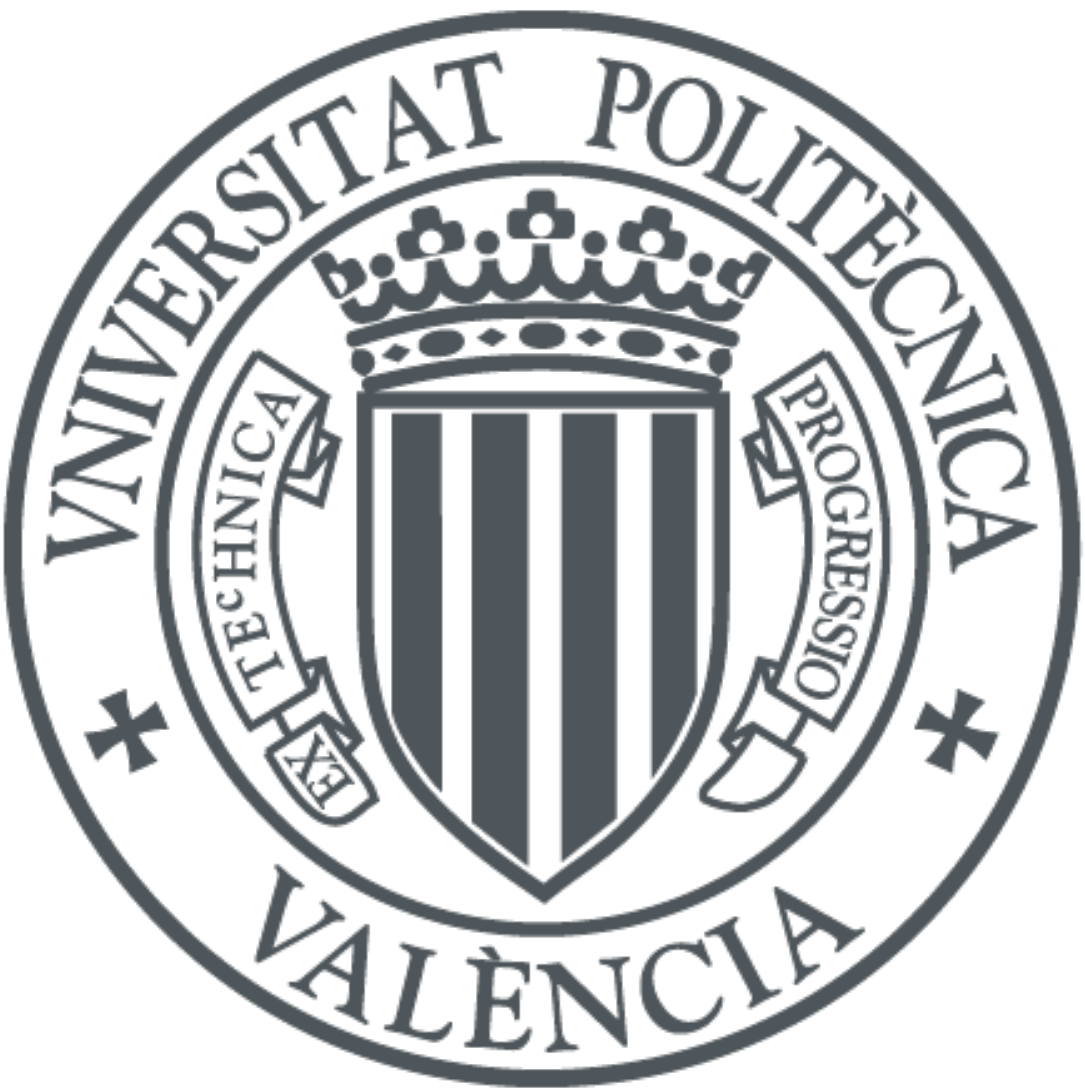

The final publication is available at

https://doi.org/10.1016/j.ymssp.2019.05.052

Copyright Elsevier

Additional Information 


\title{
Integration of intermittent measurement from in-cylinder pressure resonance in a multi-sensor mass flow estimator
}

\author{
C. Guardiola ${ }^{1}$ B. Pla ${ }^{1}$ P. Bares ${ }^{1}$ J.C. Peyton Jones ${ }^{2}$ \\ Universitat politècnica de València ${ }^{1}$ \\ Villanova University ${ }^{2}$
}

\begin{abstract}
A novel technique of trapped mass determination, based on the in-cylinder pressure resonance, has been recently published by the authors. However, the method only works when sufficient resonance intensity exists and the current formulation might preclude its implementation in real-time due to excessive computational burden.

The present paper proposes an iterative algorithm for reducing the number of operations, an adaptive filter to identify faulty measurements and a Kalman filter that combines several sensors and models, currently used in commercial light-duty engines, to ensure a continous estimation of trapped mass, air mass, and exhaust gas recirculation (EGR).

The filter is implemented using experimental data of a EURO 6 light-duty engine in a world harmonize light-duty test cycle (WLTC), showing the potential of being implemented in real driving conditions with robustness and harnessing a new measurement to improve the accuracy and response of current estimations.
\end{abstract}

Key words: Resonance, Internal combustion engines, Signal processing, observer, Kalman filter

\section{Introduction}

A new method for trapped mass determination has been recently published by the authors[1,2]. The method analyses the in-cylinder pressure oscillations created by combustion to determine the cylinder charge by assuming the gas properties at the end of the combustion. A specific transformation which

* Corresponding author:

Email address: pabamo@mot.upv.es (P. Bares $\left.{ }^{1}\right)$. 
takes into account the theoretical resonance evolution was developed to avoid time-frequency analysis [3], such as short time Fourier transform (STFT) or Wigner distributions (WD). The algorithm does not require any calibration in pent-roof combustion chambers and a single parameter for bowl-in-piston geometries. The calibration of such parameter can be derived from experimental data by relying in external sensors, e.g. air mass flow with EGR valve closed, or by using numerical simulations, as demonstrated in [4] by using finite element methods (FEM). The potential of the method has been highlighted by improving the accuracy and transient response of some models that need 15 from a trapped mass estimation. Some examples can be found in [5] for $\mathrm{NO}_{x}$ modelling, in [6] for exhaust temperature estimation, in [7] for residual gases modelling, or in [8] for knock prediction.

Nevertheless, the model needs from sufficient resonance intensity to provide the system with a robust estimation of trapped mass. The requirement of sufficient resonance might be an issue in small engines, where resonance is damped faster, and at some operating conditions where combustion is particularly smooth and slow, e.g. idle in compression ignited (CI) engines and low-load in spark ignited (SI) engines.

Furthermore, the estimation of many combustion control parameters, e.g. oxygen concentration at the intake or EGR mass flow, require from a combination of several sensors and models, which may suffer from bias and slow response, which encourages the design of sensor data fusion algorithms to ensure an accurate and robust output. Air mass flow sensors have a fast response that varies from 30-50 ms [9] but are subjected to severe ageing due to the accumulation of dust on the sensing element $[10,11,12]$, injector models suffer from minor errors in hole diameter, caused by unavoidable manufacturing variations and to deposits accumulation [13], universal exhaust oxygen sensors (UEGO) present a linear and fast response (below $100 \mathrm{~ms}$ ) over a wide range but errors above $5 \%$ have been reported after 3000 working hours [14], the measurement of EGR by gas analysers has a slow response due to gas extraction procedure and might be non-representative when gas mixing is not complete at the probe location[15, 16, 17], while onboard EGR sensing is not possible nowadays and must be estimated through the speed density method which might diverge from the real behaviour of the engine at off-design conditions, specially in transient $[18,19]$.

To combine measurements at various locations, dynamics at the intake is represented by emptying and filling model: a control volume with the mass and energy conservation equations, by assuming no wall heat transfer and perfect gas mixture composition [20, 21, 22]. Several closed-loop observers have been 
proposed to fuse sensors and models taking into account the dynamics of the engine. In engines without external gas recirculation, a bias on the volumetric ency is updated by assuming the air mass flow given from the hot-film anemometer as an input and considering the dynamics at the intake manifold [23, 24, 25]. In engines with EGR, Liu et al. and Kolmanovsky et al. proposed a similar observer but including an EGR estimation by using the orifice model at the EGR valve [26, 27], while Zhao et al. and Castillo et al. proposed an observer by considering intake and exhaust dynamics for the oxygen concentration $[28,29]$.

Kalman filters (KF) are a concrete type of closed-loop observers where the observer gains are continuously adapted to improve the convergence and accuracy of the observer [30]. Several examples can be found in literature for automotive applications: in [31], an extended Kalman filter is proposed for air-charge estimation improvements in SI engines, in [32] the complete adiabatic manifold model is used in combination of a throttle model and a first order system modelling the temperature sensor dynamics for predicting the actual in-cylinder air flow, and in [33] an intake manifold model of a CI engine with EGR is used to calculate all the mass flows.

The present paper aims to design a robust filter harnessing the trapped mass estimation obtained from resonance. Concretely a three steps procedure is proposed:

(1) An iterative calculation of the transformation for reducing the computational burden of the algorithm.

(2) An adaptive filtering to discern outliers with erratic measurements.

(3) And a Kalman filter that combines such intermittent measurement with sensors and models that can be found nowadays in a EURO 6 light-duty engine. The EGR valve is also used as an intermittent input for updating the volumetric efficiency when the EGR valve is closed.

A four-stroke compression ignited (CI) engine has been tested in specific transient tests and in a world harmonize light-duty test cycle (WLTC) to validate the proposed filter.

The paper is structured as follows: next section describes the experimental set-up and the main sensors employed, section three and four describe the algorithm proposed to calculate the trapped mass and discern faulty measurements with the resonance method and the observer that combines all sensor and models, section five shows the results of the filter for the tests recorded, and last section highlights the strengths and future work of the method. 


\section{Experimental setup}

90 A four-stroke diesel engine with 1.5-l of total displacement was used for testing the proposed filter. It is a four-cylinder engine with a turbo-charger and equipped with common rail injection system. Table 1 summarizes the main engine characteristics.

Table 1

Main engine characteristics

\begin{tabular}{lcc} 
& Units & Value \\
\hline Cylinders & {$[-]$} & 4 \\
Combustion type & {$[-]$} & CI \\
Unitary displacement $\left(V_{\text {dis }}\right)$ & {$[\mathrm{cc}]$} & 374.65 \\
Bore $(D)$ & {$[\mathrm{mm}]$} & 73.5 \\
Compression ratio & {$[-]$} & $17: 1$
\end{tabular}

95 Figure 1 shows a scheme of the engine, pointing out the main mass flows and the sensors used: A hot-film anemometer was used to measure the air mass flow passing through the compressor $\left(m_{\text {air }}\right)$, a thermocouple with $1.5 \mathrm{~mm}$ of diameter and a piezo-resistive pressure sensor were placed at the intake to measure the temperature and the pressure of the intake manifold $\left(T_{i n t}\right.$ and $\left.p_{\text {int }}\right)$, an AVL GU13P piezo-electric sensor was used to measure the in-cylinder pressure $\left(p_{c y l}\right)$, and an UEGO oxygen sensor was placed at the exhaust to measure oxygen concentration with lambda $\left(\lambda_{e x h}\right)$. Variables that cannot be measured by sensors, such as mass and oxygen concentration at the intake $\left(m_{\text {int }}\right.$ and $\left.F_{\text {int }}\right)$, mass and oxygen concentration at the exhaust $\left(m_{\text {exh }}\right.$ and $\left.F_{\text {exh }}\right)$, and 105 High-pressure EGR mass flow $\left(m_{E G R-H P}\right)$ are also shown in grey. 


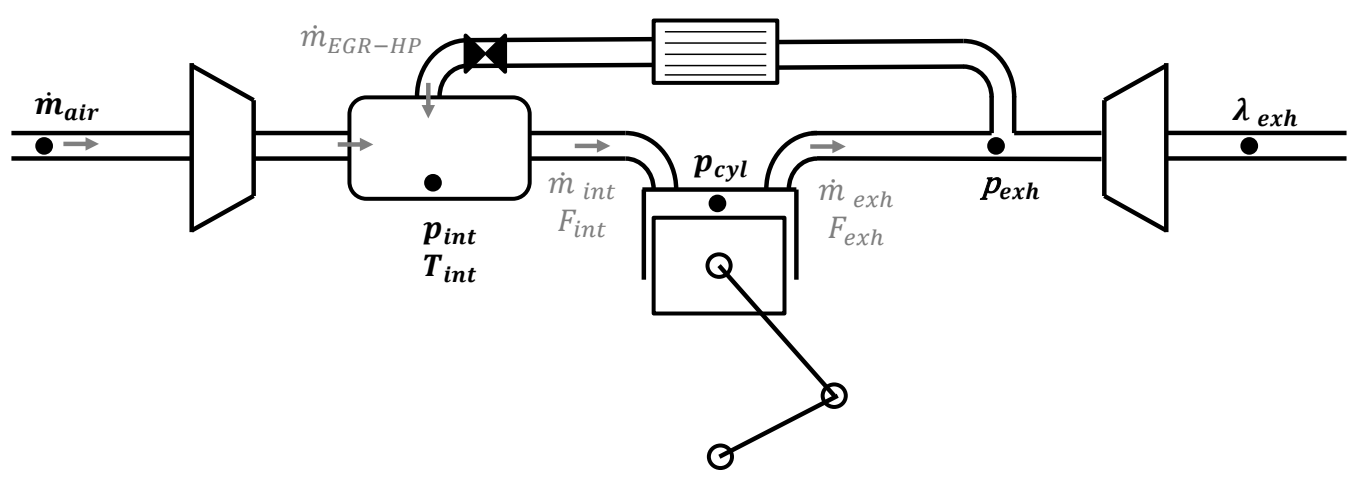

Fig. 1. Scheme of main mass flows and sensors used

Although some steady and specific transient tests will be shown for illustration purposes, the validation of the filter will be shown in a WLTP cycle in order to analyse the method in an homologation test, close to real conditions. Figure 2 shows the break mean effective pressure (BMEP) and engine speed during the WLTP cycle. Two repetitions were made: one with the EGR valve closed during the full tests and other with the default EGR valve controls during the first part of the cycle.
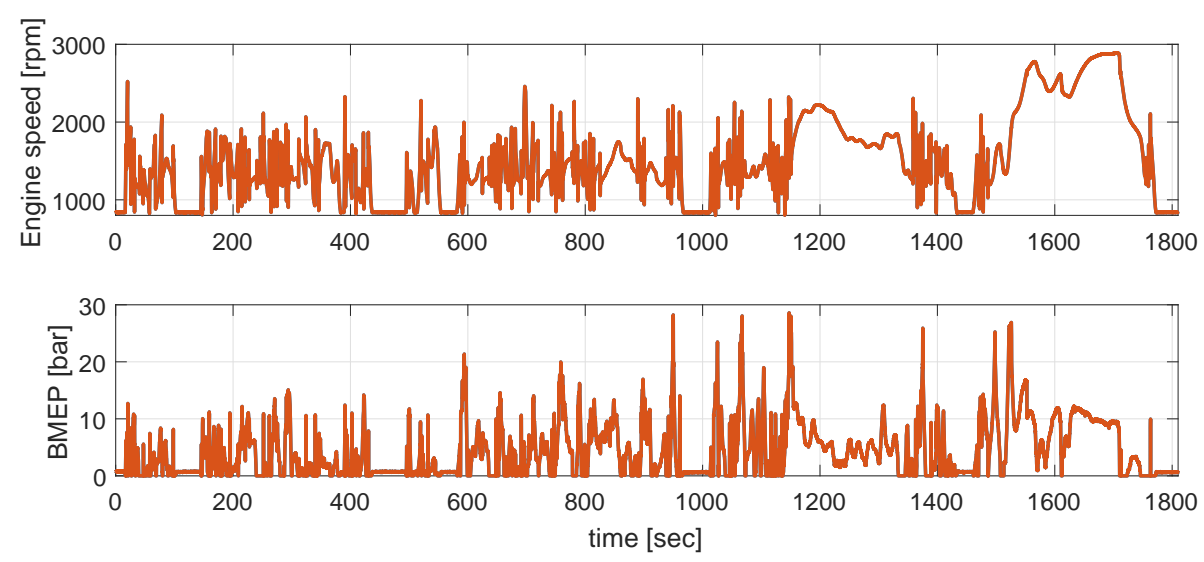

Fig. 2. WLTP cycle

\section{Trapped mass estimation}

Top plot of Figure 3 shows the in-cylinder pressure and the apparent heat release rate of a cycle at $2400 \mathrm{rpm}$ and $88 \mathrm{Nm}$ of torque. The pilot combustion, which consists in a single phase of premixed combustion, can be located between -8 and -3 crank angle degrees after the top dead center (CAD-ATDC), while the main combustion can be divided in a first premixed combustion from 
10 to 20 CAD-ATDC and a second diffusive phase, from 20 to 45 CAD-ATDC. The bottom plot shows the result of high-pass filtering the in-cylinder pressure signal at $3.5 \mathrm{kHz}$. It can be appreciated that, although the pilot injection only represented a $6.63 \%$ of the total fuel injected $(1.4$ over $21.1 \mathrm{mg} / \mathrm{str}$ ) the oscillation created is on the same range than the oscillation created by the main injection, while at the main injection event, the resonance excitation is mainly caused by the first pre-mixed autoignition, and not the later diffusive combustion. This is also coherent with the work developed by Kyrtatos et al. in CI combustions [34, 35]. A good overview of internal combustion engines fundamentals can be found in [36], while an analysis of the resonance excitation of various combustion modes has been recently presented in [37].
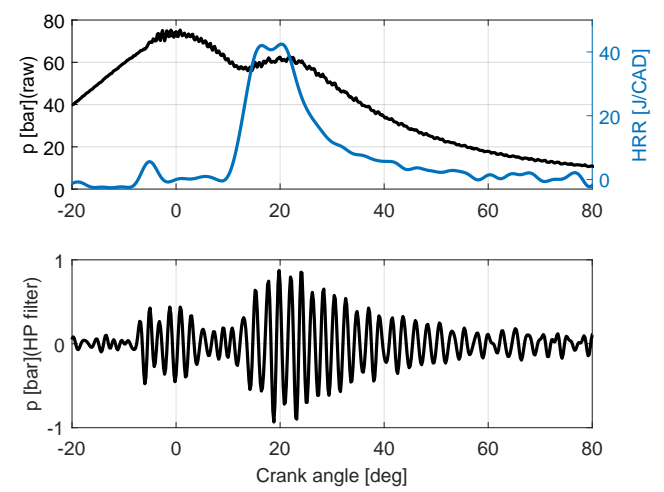

Fig. 3. In-cylinder pressure and apparent heat release rate (top plot) and high-pass filtered in-cylinder pressure of a cycle at $2400 \mathrm{rpm}$ and $88 \mathrm{Nm}$ of torque

C.S. Draper solved the wave equation by using cylindrical contour conditions and Bessel functions. He found a proportional relation between the speed of sound $(a)$ and the frequency of the in-cylinder pressure resonance $\left(f_{\text {res }}\right)$ [38], following:

$$
f_{\text {res }}=\frac{B a}{\pi D}=\frac{B \sqrt{\gamma p V}}{\pi D \sqrt{m}}
$$

where $B$ is a Bessel constant (1.842 for the first radial mode), $\gamma$ the ratio of the heat capacities, $D$ the bore of the engine, $V$ the instantaneous chamber volume, $p$ the in-cylinder pressure, and $m$ the trapped mass.

Equation (1) was obtained by assuming that the combustion chamber is cylindrical. Although this assumption can be used when the piston is far from the TDC, it is not completely true in bowl-in-piston combustion chambers when the piston approaches the TDC. The effect of the bowl can be obtained experimentally or by a finite element method simulation, such as demonstrated in 
[4]. For the present engine a $13 \%$ difference was found near the TDC $(B=2.1$ instead of 1.842) but the effect can be considered negligible after 40 CADATDC.

The authors, proposed an application in [1] and [2] of the work done by C.S.

Draper, by identifying the evolution of the resonant frequency with a timefrequency analysis (concretely the STFT), and later obtaining the trapped mass by rearranging (1), such as:

$$
m=\frac{B^{2} \gamma p V}{\left(\pi D f_{r e s}\right)^{2}}
$$

To improve the precision and reduce the number of operations of the algorithm, the authors presented a modification of the Fourier transform by including Draper's equation in the signal processing [3]. The proposed transformation converts the in-cylinder pressure in the time-domain (or crank angle based) into several pressure waves characterized by virtual masses. The real trapped mass will maximize this transformation, in the same way that a peak is obtained in a Fourier transform when processing a constant frequency harmonic.

A high-pass filter (or a band pass) is suggested to eliminate the effect of the low-frequency components which are associated with the pressure rise caused by combustion and the piston movement and not the resonance of the chamber. Furthermore, in order to analyse only the range excited by resonance a window function is also applied to the high-pass filtered pressure $p_{h p}$, as following:

$$
S(m)=\sum_{\alpha=\alpha_{2}}^{\alpha=\alpha_{1}} w\left(\alpha-\alpha_{1}\right) p_{h p}(\alpha) e^{-j 2 \pi \sum_{\phi=0}^{\phi=\alpha} T(\phi) \frac{B(\phi) \sqrt{\gamma(\phi) p(\phi) V(\phi)}}{\pi D \sqrt{m}}} T(\alpha)
$$

where $\alpha_{1}$ and $\alpha_{2}$ is the interval where the resonance analysis is performed, $w$ is a window function of $\alpha_{2}-\alpha_{1}$ length, and $T(\alpha)$ is the instantaneous sampling rate, which is constant only in time-based in-cylinder pressure acquisition or if the instantaneous engine speed fluctuations are negligible.

An appropriate window length is crucial for a robust and accurate estimation of the trapped mass. Wider windows might add noise content by including in-cylinder pressure without resonance (before combustion or when resonance is already damped), but if they are properly located, using wide windows more resonance information is included, which improves the trapped mass accuracy and facilitate the noise rejection. Figure 4 shows the result of the resonance transformation over the in-cylinder pressure signal shown in Figure 3 for three 
different lengths (20,40 and $60 \mathrm{CAD})$ using a blackman-harris window located at $20 \mathrm{CAD}$-ATDC. Here, equation 3 was processed from 300 to $1000 \mathrm{mg} / \mathrm{str}$ with a resolution of $1.4 \mathrm{mg}$ (500 samples).

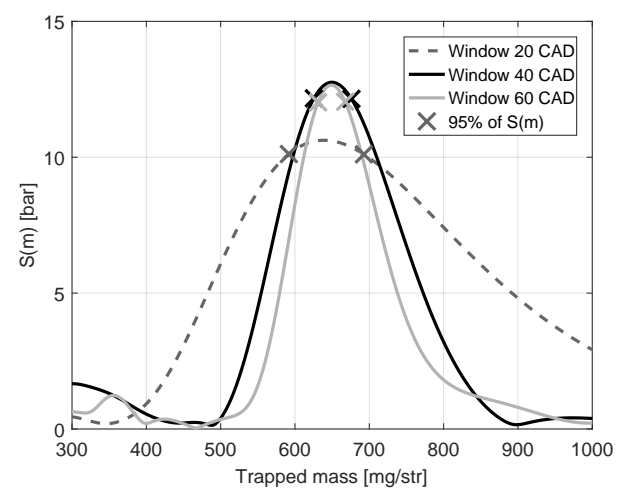

Fig. 4. Resonance transformation for trapped mass estimation proposed in [3] applied to one in-cylinder pressure cycle, working at $2400 \mathrm{rpm}$ and $88 \mathrm{Nm}$ of torque

Regarding the precision, the shape of the transformation gives an insight of how precise is the method. In this work, the mass deviation leading to an amplitude in the transformation of $95 \%$ of its maximum value $\left(S_{\max }\right)$ is considered an appropriate quality indicator $\left(I_{q}\right)$ of the precision of the method, such as:

$$
I_{q}=\Delta m_{\left[\Delta S=0.05 S_{\max }\right]}
$$

In the example shown in Figure 4 the $95 \%$ of the amplitude of $S(m)$, for each window length, has been marked with crosses: a window length of 20 CAD would have an associated precision of $\pm 50 \mathrm{mg} / \mathrm{str}$, a $40 \mathrm{CAD}$ window would have an associated precision of $\pm 25 \mathrm{mg} / \mathrm{str}$, while a $60 \mathrm{CAD}$ window would have an associated precision of $\pm 18 \mathrm{mg} / \mathrm{str}$.

\section{Iterative algorithm}

Using an equispaced grid of virtual masses to find the maximum of the function defined in Equation (3) with sufficient resolution might imply a non-admissible computational cost for a real-time implementation in an electronic control unit (ECU). In this paper an iterative fixed-point algorithm is proposed to avoid such intensive data processing.

195

The mass value for the initialization of the algorithm $\left(m_{0}\right)$ was obtained by using the speed density method, but other alternative methods might be used, 
such as using a few mass candidates with a coarse initialization grid. For completing the initialization of the algorithm, the function at the initial point and at a near location $\left(m_{0}+\Delta m\right)$ are analysed to determine if the function increases or decreases. Once the sign of the derivative is known, the iterative procedure can be started.

The new mass candidate $\left(m_{\text {new }}\right)$ is obtained by multiplying the previous mass $\left(m_{\text {old }}\right)$ by $1 \pm K$, where $K$ determines how fast the maximum of the function $S(m)$ is located. A small value of $K$ would imply small steps in mass and hence a slow convergence, but a big value of $K$ might excessively surpass the actual mass and would also slow down the number of iterations required. A good initial value of $K$ might be the expected error of the initial method, i.e. in this work, as the speed density method was used with a constant volumetric efficiency of 0.85 , a $10 \%$ variation at each step is considered, i.e. $K_{0}=0.1$.

To ensure the convergence of the algorithm, $K$ should be reduced once the maximum is surpassed, i.e. the sign of the derivative of the mass $\left(m_{\text {new }}-m_{\text {old }}\right)$ changes. If $K$ is reduced too fast, the algorithm might last many iterations to reach again the maximum, but if $K$ is reduced smoothly, the algorithm might be going from one side to the other side of the peak without a proper convergence. In the presented algorithm a reducing factor $\left(k_{r e d}\right)$ of 0.33 is proposed, i.e. $K$ is divided by three each time the maximum is reached.

Regarding the window length used for the function $S(m)$ : wide windows have a more concise spectrum, which might induce to errors if the initial trapped mass estimation is far away from the real trapped mass, henceforth, small windows are preferred at the first iterations, while wider windows might be used at the final steps. In the designed algorithm the initial window is relatively small (20 CAD) and is increased each time that the maximum is surpassed, until a maximum length (60 CAD) is reached.

The convergence criteria of the iterative algorithm is smaller mass variations than a given algorithm resolution $\left(\varepsilon_{m}\right)$, but a maximum of 100 iterations is also imposed. Figure 5 summarizes the implemented algorithm. 


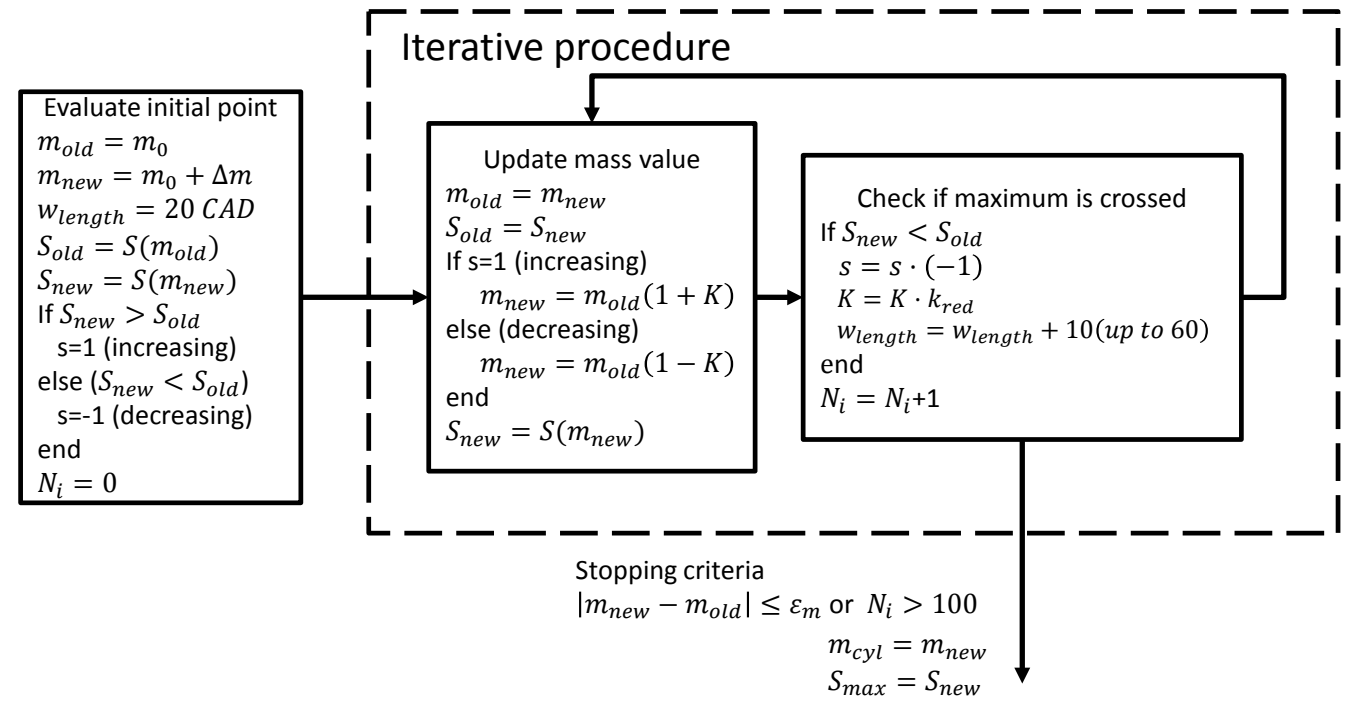

Fig. 5. Scheme of the iteration method proposed to find the actual trapped mass

where $s$ is the state (increasing or decreasing mass).

Figure 6 shows the result of the iterative method in the cycle shown in figure 3 for an initial mass assumption with a $20 \%$ error. The iterations, marked with crosses, have been represented with various colors indicating the window length used. Note how the window length is increased and the mass steps are being reduced in order to converge to the final solution.

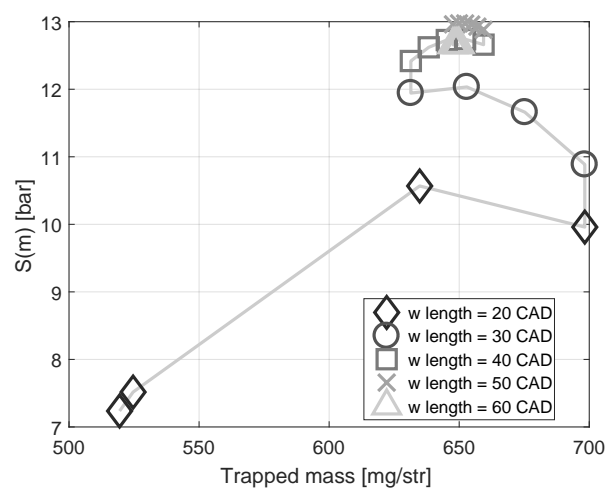

Fig. 6. Mass and amplitude results for an iteration starting with an initial error of $20 \%$ of the actual trapped mass

The parameters chosen for the iterative method, namely $K$ and $k_{\text {red }}$, ensure a fast and robust convergence, even in case of an initial mass guess with a significant bias: Figure 7 shows the convergence of the algorithm for various initial masses with errors ranging from $-40 \%$ to $40 \%$. The algorithm lasts between 
18 and 25 iterations to locate the maximum within $1 \mathrm{mg}$ resolution, while a 6 mg confidence $\left(\varepsilon_{m}<1 \%\right)$ is achieved before 15 iterations.
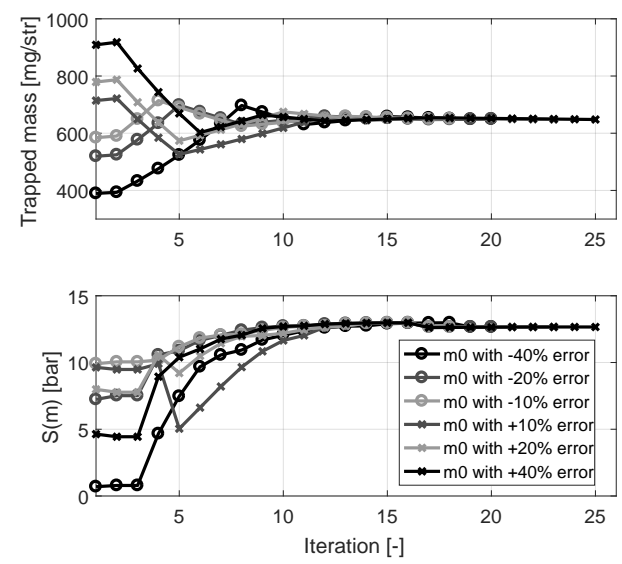

Fig. 7. Convergence of the algorithm for various values of initial mass

The iterative method only gives few points of the transformation at each window length, thus complicating the estimation of a method precision indicator based on the shape of the peak. Nevertheless, the peaks can be described by a quadratic function, following:

$$
S(m)=S_{\max }-k_{q}\left(m-m_{\max }\right)^{2}
$$

and $k_{q}$ can be found with the second derivative of the the function, such as:

$$
\frac{\mathrm{d}^{2} S(m)}{\mathrm{d} m^{2}}=2 k_{q}
$$

In the discrete domain the peaks are fully described with three points, following:

$$
\begin{gathered}
{\left[\frac{\mathrm{d} S(m)}{\mathrm{d} m}\right]_{m=\frac{m_{k+1}+m_{k}}{2}} \approx \frac{S_{k+1}-S_{k}}{m_{k+1}-m_{k}}} \\
{\left[\frac{\mathrm{d}^{2} S(m)}{\mathrm{d} m^{2}}\right]_{m=\frac{m_{k+1}+m_{k}}{2}} \approx \frac{\left[\frac{\mathrm{d} S(m)}{\mathrm{d} m}\right]_{k+1}-\left[\frac{\mathrm{d} S(m)}{\mathrm{d} m}\right]_{k}}{m_{k+1}-m_{k}}}
\end{gathered}
$$

255 Figure 8 has separated the points shown in Figure 6 with different window length. At each subplot, the points used have been represented with crosses, 
the final function modelled by (5) has been plotted with a continuous line, and the actual function obtained with a equispaced grid of virtual masses was represented with a dashed line. Here, $k_{q}$ has been found by using the three last points at each iteration step.
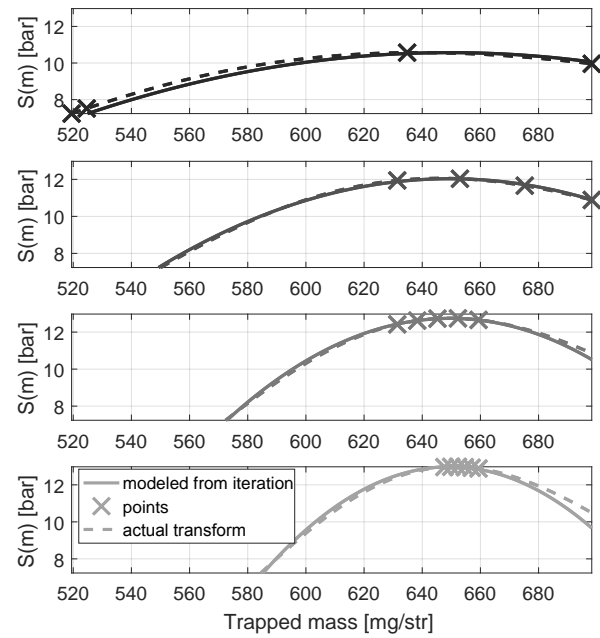

Fig. 8. Model estimation of quadratic peak for an iteration event separating point with different window lengths

It must be noticed that using only three points the shape of the function is captured with sufficient precision to provide the system with an estimation of the quality indicator. Concretely, the mass variation required to lower the amplitude to $95 \%$ of the maximum value can directly obtained through:

$$
I_{q}=\Delta m_{\left[\Delta S=0.05 S_{\max }\right]}=\sqrt{\frac{0.05 S_{\max }}{k_{q}}}
$$

Table 2 shows the results of the index quality using three points with the quadratic assumption for the six initial masses shown in Figure 7. Note that although the points used for the calculation have changed, the method is obtaining a similar value of the quality metrics at each window length. 
Table 2

Estimated mass length for $95 \%$ of the maximum amplitude for various iteration events $\left(I_{q}\right)$

\begin{tabular}{l|cccccc} 
Window & $m_{0}-40 \% \varepsilon_{m}$ & $m_{0}-20 \% \varepsilon_{m}$ & $m_{0}-10 \% \varepsilon_{m}$ & $m_{0}+10 \% \varepsilon_{m}$ & $m_{0}+20 \% \varepsilon_{m}$ & $m_{0}+40 \% \varepsilon_{m}$ \\
\hline 60 CAD & 50.9740 & 49.6127 & 48.9297 & 47.5167 & 57.7301 & 58.7931 \\
$50 \mathrm{CAD}$ & 35.3174 & 35.4807 & 34.5190 & 33.7420 & 35.5127 & 34.1561 \\
40 CAD & 25.9756 & 26.0884 & 26.0718 & 26.1513 & 26.1633 & 25.8334 \\
30 CAD & 21.7032 & 21.6002 & 21.7915 & 21.6403 & 21.6501 & 21.7641
\end{tabular}

\section{Adaptive filtering}

Some combustion events do not sufficiently excite resonance to estimate the trapped mass with reliability, e.g. idle. The amplitude of the resonance transformation is a good indicator and can be used to directly discard most of the erratic measurements. Figure 9 shows the result of the resonance transform in part of the WLTC test (5000 cycles): the amplitude of the transformation has been represented in the color-scale, being lighter colors, cycles related with more intense pressure oscillations.

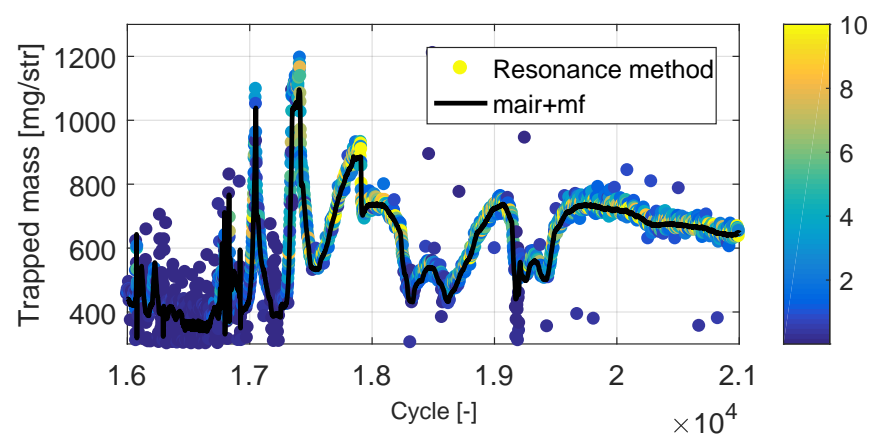

Fig. 9. Trapped mass estimation from sensors and from the resonance method for 5000 cycles in a WLTP with the EGR valve closed (the amplitude of the resonance transformation is represented by the colorscale)

The effect of removing cycles with low intensity is highlighted in Figure 10 where all the cycles with a resonance amplitude below 0.5 bar have been represented with a different color. Note that most of the cycles with sufficient resonance are coherent with the value obtained from sensors. 


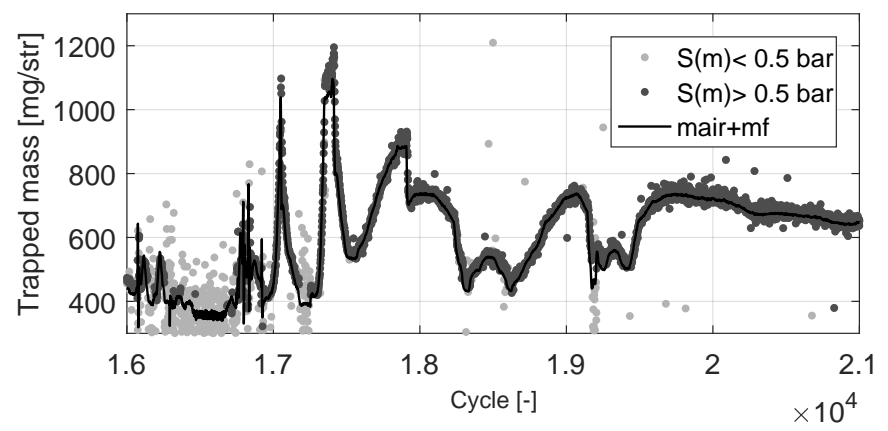

Fig. 10. Sensor measurement and result of the resonance method for 5000 cycles in a WLTP with the EGR valve closed (points with low amplitude have been coloured with light gray)

Nevertheless, the method can give erratic measurements at some cycles when other phenomena are confused with the first radial model of resonance $(B=$ 1.842), two of the most common interferences are:

- Combustion: The representative frequencies of normal combustion are commonly located below $2 \mathrm{kHz}$ [39], and they are easily eliminated with a highpass filter. Nevertheless, at fast combustions, they might also exhibit high frequencies which can be confused with resonance, creating peaks in $S(m)$ at higher values of virtual masses than the actual one.

- Other resonant modes: The first radial mode corresponds to $B=1.842$, while the second one to $B=3.05$, so they can be properly separated with a proper band pass or using the appropriate initial mass for iteration, as the second mode gives a mass 2.73 times smaller than the actual one. However, because of aliasing, higher resonant modes can be associated to lower frequency components if the sampling frequency does not reach the Nyquist criteria $\left(F_{s}>=2 f_{\text {res }}\right)$, and hence, creating virtual masses around the whole spectrum.

An adaptive filter has been designed to reject outliers when the value obtained from the resonance method $\left(m_{c y l}\right)$ is far away from the expected value. The rejection of the outliers is based on intake pressure variations and an estimation of the cycle-to-cycle trapped mass variations $\left(\sigma_{m}\right)$. The adaptive filter consists on updating the cycle-to-cycle trapped mass variations with an infinite impute response (IIR) filter, characterized by $k_{f}$, as follows:

$$
\sigma_{m}^{k}=k_{f} \sigma_{m}^{k-1}+\left(1-k_{f}\right)\left|m_{c y l}^{k}-m_{f i l t}^{k-1}\right|
$$

where $m_{c y l}^{k}-m_{f i l t}^{k-1}$ is used to update the cycle-to-cycle variability and $m_{f i l t}$ is the trapped mass filtered value. In the final work $m_{f i l t}$ is given by the observer that will be defined later. The value of $k_{f}$ comprised between 0 and 1 , must be chosen to ensure a fast adaptation of $\sigma_{m}$ when operating conditions are 
varied but rejecting the noise caused by outliers. In the present work a value of 0.9 has been selected.

The trapped mass measurement $m_{c y l}^{k}$ is accepted only if:

$$
\hat{m}_{c y l}^{k} \epsilon\left[m_{c y l}^{k-1} \frac{p_{i n t}^{k}}{p_{i n t}^{k-1}}-k_{\sigma} \sigma_{m}^{k}, m_{c y l}^{k-1} \frac{p_{i n t}^{k}}{p_{i n t}^{k-1}}+k_{\sigma} \sigma_{m}^{k}\right]
$$

where $k_{\sigma} \sigma_{m}^{k}$ is the margin related to cycle-to-cycle trapped mass variations. Note that in order to consider cycle-to-cycle trapped mass variations caused by sharp intake mass flow changes, e.g. variable geometry turbine (VGT) steps, intake pressure $\left(p_{\text {int }}\right)$ variations are used to estimate the increase at the intake mass flow, such as:

$$
m_{\text {int }}^{k} \approx m_{\text {int }}^{k-1} \frac{p_{i n t}^{k}}{p_{i n t}^{k-1}}
$$

Here, the intake temperature $\left(T_{\text {int }}\right)$, the fuel mass $\left(m_{\text {fuel }}\right)$ and the volumetric efficiency $\left(\eta_{v}\right)$ variations are not considered.

In Equation (11), $k_{\sigma}$ defines the margin for measurement acceptance. Here, outliers are rejected when the trapped mass variations are above five times the expected cycle-to-cycle variability $\left(k_{\sigma}=5\right)$.

Figure 11 illustrates the outliers identification procedure over a VGT step, with the engine working at $1250 \mathrm{rpm}$ and $60 \%$ load. In this example, the filtered trapped mass has been obtained by using another IIR filter over the trapped mass estimation $\left(m_{c y l}\right)$. Note that all the raw measurements (even outliers) are used for the adaptation of $\sigma_{m}$ as it is required to contemplate sudden changes in the trapped mass or different cycle-to-cycle dispersion, while the update of the filtered trapped mass does not take into account the identified outliers for its calculation. 


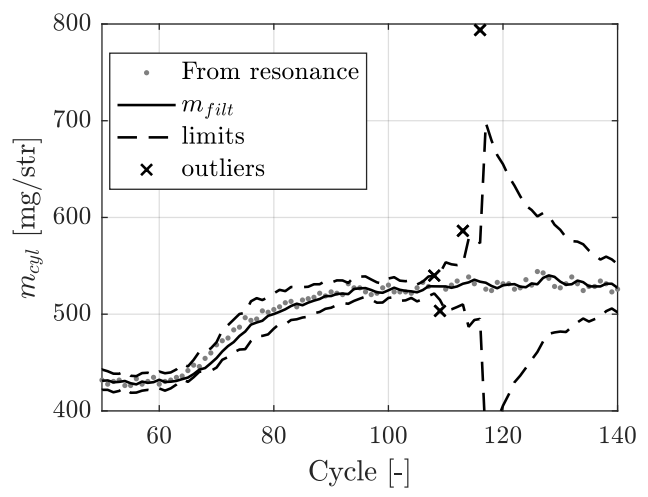

Fig. 11. Outliers identification procedure in a VGT step working at $1250 \mathrm{rpm}$ and $60 \%$ load

In order to consider the accumulation effects at the intake manifold its dynamics must be considered. In this engine, an inter-cooler lowers the EGR temperature close to the temperature of the gases at the intake, consequently, the isothermal model was used, which is defined by:

$$
p_{\text {int }}^{k+1}=p_{\text {int }}^{k}+\frac{T_{i n t}^{k} R}{V_{m}}\left(m_{\text {air }}^{k}+m_{E G R}^{k}-m_{\text {int }}^{k}\right)
$$

where $R$ is the gas constant, $V_{m}$ the intake manifold volume, and $m_{\text {int }}$ the intake mass flow, which can be computed by using the volumetric efficiency, through: 


$$
m_{i n t}^{k}=\eta_{v}^{k} \frac{p_{i n t}^{k} V_{d i s}}{R T_{i n t}^{k}}
$$

340

The intake manifold volume has been inferred by performing VGT steps with the EGR valve closed. Figure 12 shows the VGT step performed at $1250 \mathrm{rpm}$ and $60 \%$ load, where the intake pressure was varied from 1.16 to 1.45 and back to 1.16 bar. The isothermal model represented by a black line was feed with the fitted value of intake manifold volume, which was $1.89 \mathrm{l}$.

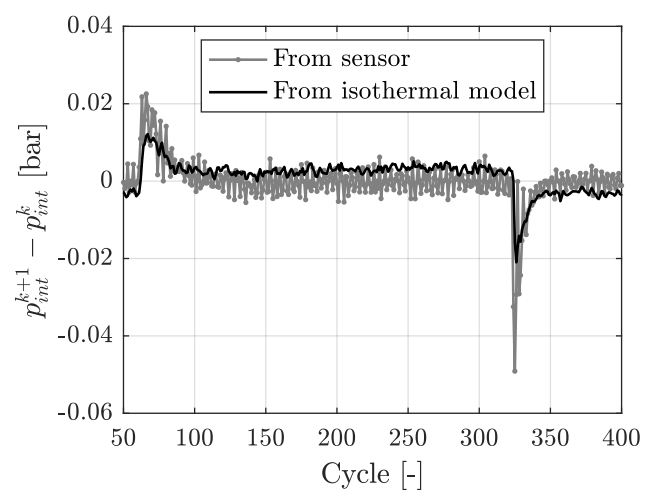

Fig. 12. Intake pressure variations in a VGT step (from 1.16 to 1.45 and back to 1.16 bar), working at $1250 \mathrm{rpm}$ and $60 \%$ load

The system is fully defined with four states:

$$
\begin{aligned}
\theta_{\eta}^{k+1} & =\theta_{\eta}^{k} \\
\theta_{\text {air }}^{k+1} & =\theta_{\text {air }}^{k} \\
m_{E G R}^{k+1} & =m_{E G R}^{k} \\
p_{\text {int }}^{k+1} & =p_{\text {int }}^{k}+\frac{R T_{\text {int }}^{k}}{V_{m}}\left(m_{\text {air }}^{k}+\theta_{\text {air }}^{k}+m_{E G R}^{k}-\left(\eta_{v}^{k}+\theta_{\eta}^{k}\right) \frac{p_{\text {int }}^{k} V_{\text {dis }}}{R T_{\text {int }}^{k}}\right)
\end{aligned}
$$

And the trapped mass can be obtained by combining the intake mass flow with the injected fuel mass (obtained through an injector model or by using an UEGO sensor at the exhaust), following:

$$
m_{c y l}^{k}=\left[m_{f u e l}^{k}+\left(\eta_{v}^{k}+\theta_{\eta}^{k}\right) \frac{p_{i n t}^{k} V_{d i s}}{R T_{i n t}^{k}}\right]
$$

An extended Kalman filter (EKF) was designed to update the observer gain in order to obtain an optimal solution of the system. A discrete form with one cycle step was chosen, following the state space representation of the system: 


$$
\begin{aligned}
x_{k+1} & =f\left(x_{k}, u_{k}\right)+w_{k} \\
y_{k} & =g\left(x_{k}, u_{k}\right)+v_{k}
\end{aligned}
$$

where $x$ are the states, modelled by $f\left(x_{k}, u_{k}\right), y$ the outputs described by $355 g\left(x_{k}, u_{k}\right), u$ the inputs, $w$ the associated noise to the states equation, and $v$ the noise associated to the outputs. Concretely:

$$
x=\left(\begin{array}{c}
\theta_{\eta} \\
\theta_{\text {air }} \\
m_{E G R} \\
p_{\text {int }}
\end{array}\right), y=\left(\begin{array}{c}
m_{\text {cyl }} \\
u_{E G R} \\
p_{\text {int }}
\end{array}\right), u=\left(\begin{array}{c}
m_{\text {air }} \\
m_{\text {fuel }} \\
T_{\text {int }} \\
\eta_{v}
\end{array}\right)
$$

Here, the valve control $\left(u_{E G R}\right)$ is used to force EGR mass flow to zero when the EGR valve is closed. The KF of the state vector is defined by:

$$
\begin{aligned}
\hat{x}_{k \mid k-1} & =f\left(\hat{x}_{k-1}, u_{k}\right) \\
e_{k} & =y_{k}-g\left(\hat{x}_{k \mid k-1}, u_{k}\right) \\
\hat{x}_{k} & =\hat{x}_{k \mid k-1}+K_{k} e_{k}
\end{aligned}
$$

The KF is characterized for minimizing the expected estimation error by solving an iterative Riccati matrix equation and updating the value of the Kalman gain $(K)$. Following:

$$
\begin{aligned}
P_{k \mid k-1} & =\left(F_{k} P_{k-1} F_{k}^{T}+Q_{k}\right) \\
K_{k} & =P_{k \mid k-1} H_{k}^{T}\left(H_{k} P_{k \mid k-1} H_{k}^{T}+R_{k}\right)^{-1} \\
P_{k} & =\left(I-K_{k} H_{k}\right) P_{k \mid k-1}
\end{aligned}
$$

where $w$ and $v$ are modelled as a Gaussian distribution with zero mean and covariance matrices $Q_{k}$ and $R_{k}$, respectively, which are constant and diagonal. $F_{k}$ and $H_{k}$ are the linear state matrices representing Equations (20) and (21). As these equations are non-linear an extended Kalman filter (EKF) was used by linearising them, such as: 


$$
\begin{gathered}
F_{k, i j}=\left.\frac{\mathrm{d} f_{i}}{\mathrm{~d} x_{j}}\right|_{x=\hat{x}_{k}}=\left(\begin{array}{cccc}
1 & 0 & 0 & 0 \\
0 & 1 & 0 & 0 \\
0 & 0 & 1 & 0 \\
-\frac{V_{d i s} p_{i n t}^{k}}{V_{m}} \frac{R T_{i n t}^{k}}{V_{m}} & \frac{R T_{i n t}^{k}}{V_{m}} 1-\frac{V_{d i s}\left(\eta_{v}^{k}+\theta_{\eta}^{k}\right)}{V_{m}}
\end{array}\right) \\
H_{k, i j}=\left.\frac{\mathrm{d} g_{i}}{\mathrm{~d} x_{j}}\right|_{x=\hat{x}_{k}}=\left(\begin{array}{cccc}
\frac{p_{i n t}^{k} V_{d i s}}{R T_{i n t}^{k}} & 0 & 0 & \frac{\left(\eta_{v}^{k}+\theta_{\eta}^{k}\right) V_{d i s}}{R T_{i n t}^{k}} \\
0 & 0 & 1 & 0 \\
0 & 0 & 0 & 1
\end{array}\right)
\end{gathered}
$$

Note that inputs $(u)$ and outputs $(y)$ are indistinctly measurements or estimation of models. The formulation of the observer has been designed to tune the response of the Kalman filter by including noise at each equation.

Furthermore, the noise related to the trapped mass and EGR measurements $\left(R_{1,1}, R_{2,2}\right)$ are varied online:

- $R_{1,1}$ is changed to a high value $(\approx \infty)$ when a failure in the resonance method has been identified, i.e. the measurement is discarded, and it is set to $I_{q}$ if the estimation is accepted, i.e. the noise depends on the transformation. For avoiding failures in the peak detection method, $R_{1,1}$ has been bounded between 5 and $50 \mathrm{mg} / \mathrm{str}$.

380

- $R_{2,2}$ is set to 0 when the EGR valve is closed, i.e. EGR is certainly 0 , and it is changed to a high value $(\approx \infty)$ when it is opened, i.e. the measurement is not used.

The observer has been validated by filtering the output of the resonance 385 method in a WLTP cycle. Table 3 collects the values used in the present work for matrices $Q$ and $R$.

\section{Results}

The complete procedure has been applied to the trapped mass estimation of the resonance method in a WLTP cycle. Figure 13 shows the result of the filter in the cycle tested with the EGR valve closed: top plot shows the trapped mass output of the filter which is compared with the accepted measurements of the 
Table 3

Noise $(Q$ and $R$ ) suggested for the Kalman filter: the last column gives dimensionless value of the noise by using the average value in the test

\begin{tabular}{cccc} 
& Variable & Value [unit] & Dimensionless [\%] \\
\hline$Q_{1,1}$ & $\theta_{\eta}$ & $0.005[-]$ & 0.54 \\
$Q_{2,2}$ & $\theta_{\text {air }}$ & $1[\mathrm{mg} / \mathrm{str}]$ & 0.42 \\
$Q_{3,3}$ & $m_{E G R}$ & $10[\mathrm{mg} / \mathrm{str}]$ & 5.5 \\
$Q_{4,4}$ & $p_{\text {int }}$ & $100[\mathrm{mbar}]$ & 0.91 \\
$R_{1,1}$ & $m_{\text {cyl }}$ & $5-50($ or $\infty)[\mathrm{mg} / \mathrm{str}]$ & $1.1-11$ \\
$R_{2,2}$ & $u_{E G R}$ & $0($ or $\infty)[\mathrm{mg} / \mathrm{str}]$ & 0 \\
$R_{3,3}$ & $p_{\text {int }}$ & $10[\mathrm{mbar}]$ & 0.0091
\end{tabular}

resonance method, medium plot shows the volumetric efficiency correction, and bottom plot shows the bias correction at the air mass flow anemometer to reach such value of trapped mass. 

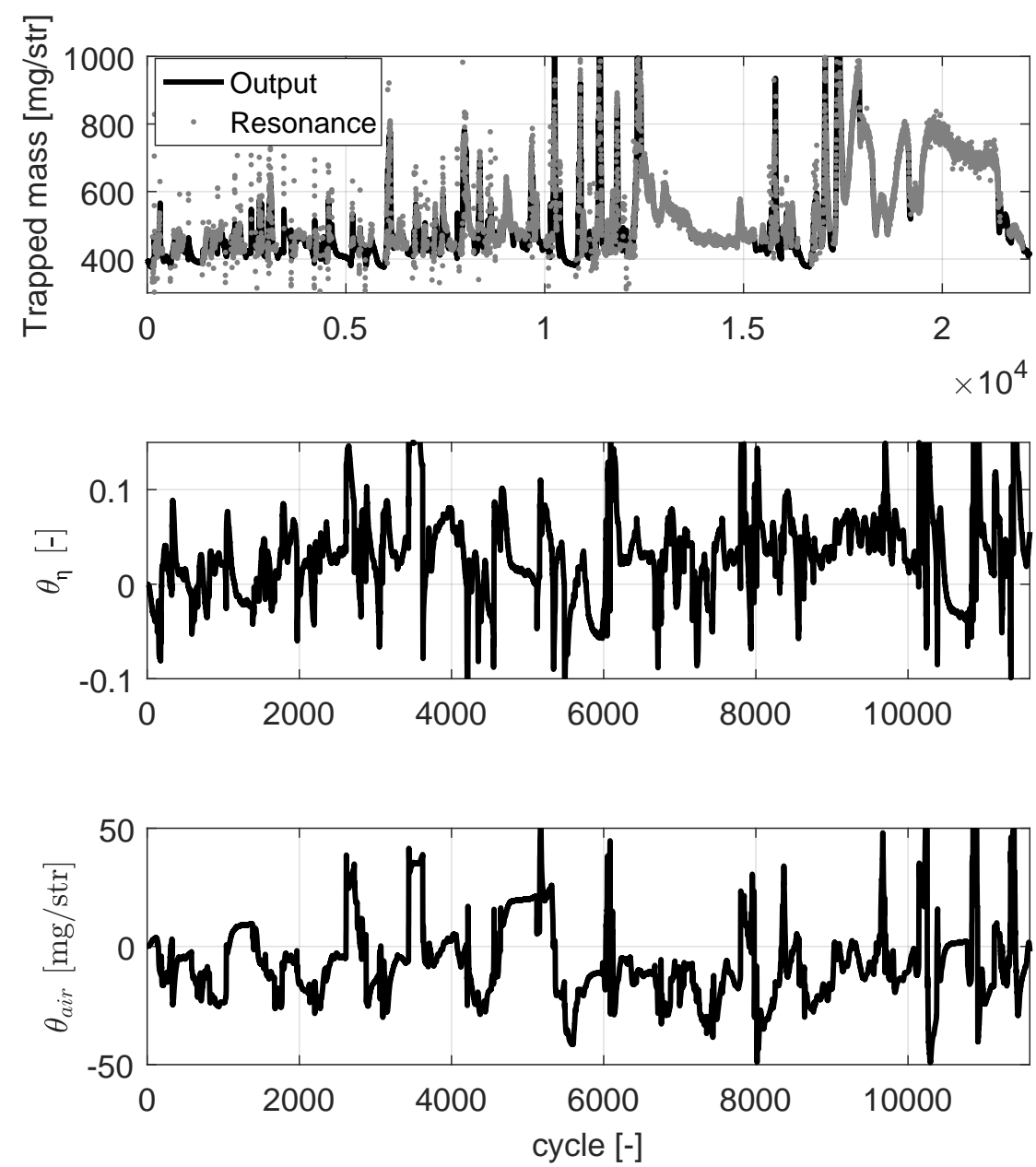

Fig. 13. WLTP cycle with the EGR valve closed: trapped mass (top plot), volumetric efficiency bias correction (medium plot), and air mass flow bias correction (bottom plot)

The volumetric efficiency has been set as a constant value which would be continuously adapted to achieve the actual trapped mass given by the resonance method. A 2D open loop model could be included to improve the Kalman filter innovation when changing the operating conditions, but in this tests a constant value was preferred to analyse the observer under the worst estimation. Note that the volumetric efficiency varies between $\pm 10 \%$ which is consistent with experimental data. The air mass flow given by the hot-film anemometer was corrected by $\pm 40 \mathrm{mg} / \mathrm{str}$ (3.18\% in average), which is also consistent given the range of operating conditions and sharp variations tested.

Figure 14 shows 100 cycles where the air mass flow has been sharply varied. 
The sum of air and fuel mass from hot film anemometer and injection model has been plotted together with the output of the filter and the trapped mass estimation from resonance. It must be noticed that resonance gives a direct estimation in the in-cylinder combustion chamber while the air mass flow sensor is located at the beginning of the intake manifold, and thus, intake manifold dynamics must be considered. The observer proposed takes into account both measurements to provide the system with an adequate transient estimation.

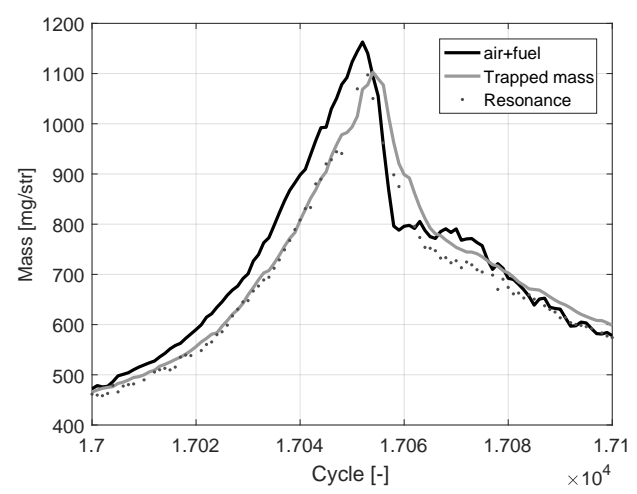

Fig. 14. Zoom over a sharp variation of air mass flow at cycle 17000

Figure 15 shows the output of the filter for the first 12500 cycles of the WLTP cycle when the EGR valve was controlled to fulfil EURO $6 \mathrm{NO}_{x}$ regulation. The estimation of the trapped mass was used to correct the volumetric efficiency (in the same way that when the EGR valve was closed), but as the Kalman filter has released one extra degree of freedom (the EGR mass flow), the corrections at the hot-film anemometer were nearly negligible $( \pm 1 \%)$, while the EGR mass flow was continuously estimated from the trapped mass, the air mass flow and the intake pressure to follow the isothermal model of the intake manifold dynamics. 

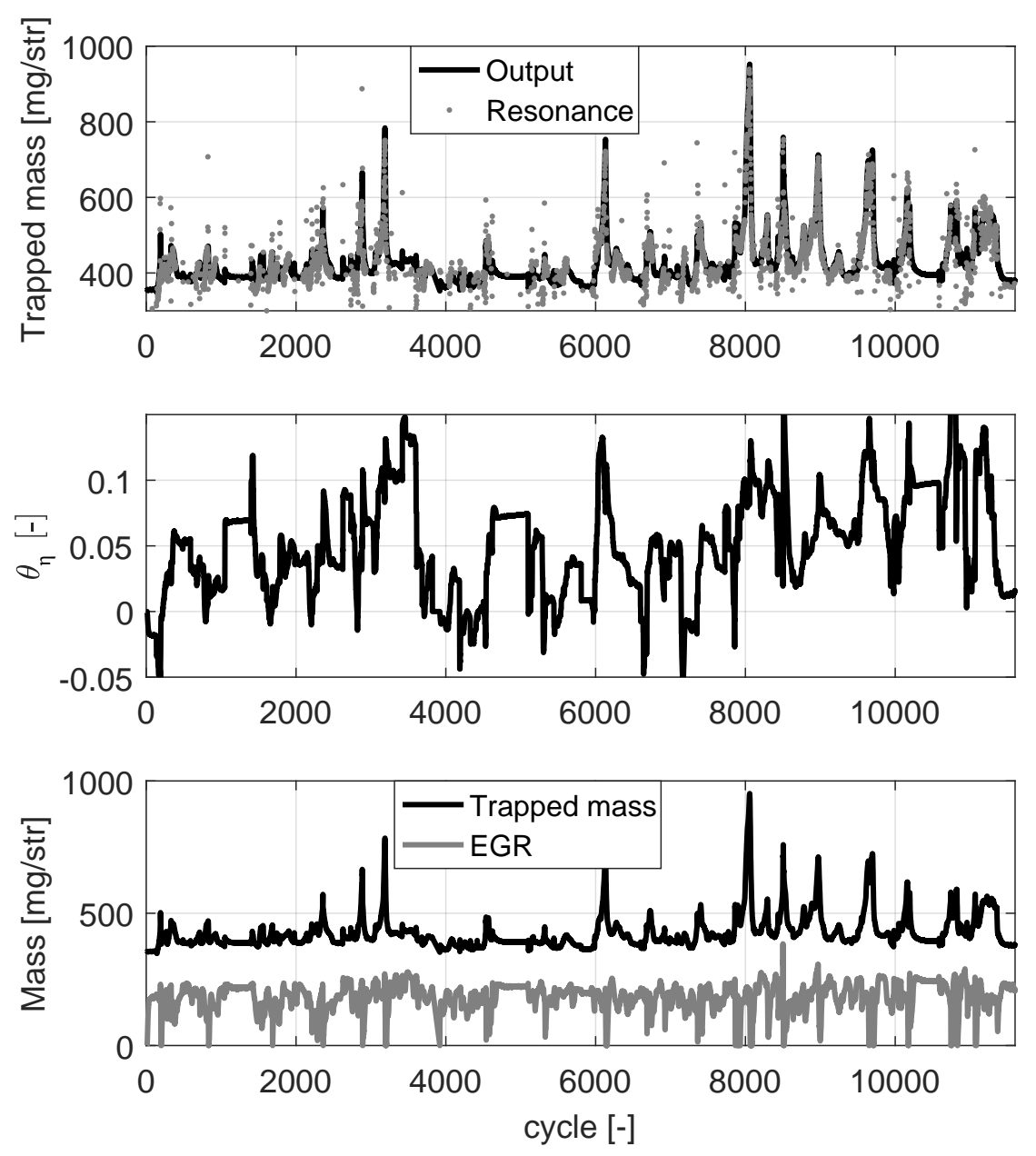

Fig. 15. WLTP cycle with EGR activated: trapped mass (top plot), volumetric efficiency bias correction (medium plot), and EGR mass flow estimated (bottom plot)

In this test the volumetric efficiency range is also on a normal range while the estimated EGR, from 0 to $50 \%$, does agree with the value expected in such conditions. Indeed, the observer provides the system with an online transient estimation of EGR which is unaffordable with the current set of sensors installed in commercial vehicles. Nowadays, EGR is computed from the speed density method, but the volumetric efficiency is stored in 2D tables and does not represent a direct feedback of the engine, as open-loop models might be affected by ageing or other non-modelled phenomena. 
The authors have recently published a method for trapped mass estimation by analysing in-cylinder pressure resonance. However, the method fails when there is no sufficient resonance intensity and the computational burden might preclude its use in future ECUs.

440

The present paper proposes an iterative algorithm for reducing the computational cost, an adaptive filtering for detecting method failures and an observer for ensuring a continuous estimation when the method fails.

- The iterative algorithm is based on a fixed point iteration by modifying the range analysed at each step. The new algorithm reduces the number of operations from 500 to 20 for the same trapped mass resolution (25 times faster). The shape of the peak, which gives an indicator of the method precision, is obtained by assuming a quadratic behaviour at the peak.

450

- The adaptive filter directly discards cycles with low resonance intensity by analysing the amplitude of the resonance transformation and detects outliers by updating a trapped mass uncertainty with an IIR filter and using the intake pressure variation for computing the trapped mass confidence interval.

- Finally, an observer has been designed to combine several sensors commonly found in commercial vehicles: air mass flow sensor, intake temperature and pressure sensors, and a lambda or an injector model for fuel estimation, by considering the intake manifold dynamics.

The volumetric efficiency is updated with the trapped mass estimation (when it exist). The hot-film anemometer can be corrected when the EGR valve is closed and its output is used to estimate the EGR in the rest of the cycles.

465

The complete procedure has been applied to experimental data in two tests of WLTP cycle: by closing the EGR valve during the full test and by using the ECUs EGR control during the first part of the WLTP cycle.

The method ensures a continuous estimation of trapped mass, air mass flow, and EGR, by updating the volumetric efficiency, by estimating the EGR, and by correcting a bias for the air mass flow measurement. The volumetric efficiency was varied $\pm 10 \%$ in both tests, the air mass flow sensor was corrected 
in $3.18 \%$ when the EGR valve was closed, and EGR was estimated between 0 and $50 \%$ in the part of the test where it was controlled. Unfortunately, the set of sensors used in the tests do not offer an adequate fast estimation of EGR for comparison, future tests would be focused on using fast gas analysers in order to validate the transient response of the observer.

\section{References}

[1] C. Guardiola, B. Pla, D. Blanco-Rodriguez, and P. Bares. Cycle by cycle trapped mass estimation for diagnosis and control. SAE Int. J. Engines, $7(3), 2014$.

[2] J. M. Luján, C. Guardiola, B. Pla, and P. Bares. Estimation of trapped mass by in-cylinder pressure resonance in HCCI engines. Mechanical Systems and Signal Processing, 66-67:862-874, 2015.

[3] A. Broatch, C. Guardiola, B. Pla, and P. Bares. A direct transform for determining the trapped mass on an internal combustion engine based on the in-cylinder pressure resonance phenomenon. Mechanical Systems and Signal Processing, 62:480-489, 2015.

[4] A. Broatch, C. Guardiola, P. Bares, and F.D. Denia. Determination of the resonance response in an engine cylinder with a bowl-in-piston geometry by the finite element method for inferring the trapped mass. International Journal of Engine Research, 2015.

[5] C. Guardiola, V. Triantopoulos, P. Bares, S. Bohac, and A. Stefanopoulou. Simultaneous estimation of intake and residual mass using in-cylinder pressure in an engine with negative valve overlap. IFACPapersOnLine, 49(11):461-468, 2016.

[6] C. Guardiola, J. Martín, J. Pla, and P. Bares. Cycle by cycle NOx model for diesel engine control. Applied Thermal Engineering, 110:10111020, 2017.

[7] C. Guardiola, V. Triantopoulos, P. Bares, S. Bohac, and A. Stefanopoulou. Simultaneous estimation of intake and residual mass using in-cylinder pressure in an engine with negative valve overlap. IFACPapersOnLine, 49(11):461-468, 2016.

[8] P. Bares, D. Selmanaj, C. Guardiola, and C. Onder. Knock probability estimation through an in-cylinder temperature model with exogenous noise. Mechanical Systems and Signal Processing, 98:756-769, 2018.

[9] K. Xu, J. Zhang, X. Wang, Q. Teng, J. Tan, and Y. Zhang. Improvements of nonlinear dynamic modeling of hot-film MAF sensor. Sensors and Actuators, A: Physical, 147(1):34-40, 2008.

[10] J. Zhao and J. Wang. Engine mass airflow sensor fault detection via an adaptive oxygen fraction observer. In 2014 American Control Conference, pages 1517-1522. IEEE, 2014. 
24 Per Andersson. Intake air dynamics on a turbocharged SI-engine with wastegate. Department of Electrical Engineering, Linköping University, 2002.

[25] E. Hendricks, T. Vesterholm, and S. C. Sorenson. Nonlinear, closed loop, 
si engine control observers. SAE Technical Papers, 1992.

[26] Y. Liu and Zhangsaifei. Measurement and diagnostic system for crankshaft of diesel engine. In ICCASM 2010 - 2010 International Conference on Computer Application and System Modeling, Proceedings, volume 13, pages V13370-V13371, 2010.

[27] I. Kolmanovsky, J. Sun, M. Druzhinina, and M. van Nieuwstadt. Charge control for direct injection spark ignition engines with egr. In Proceedings of the American Control Conference, volume 1, pages 34-38, 2000.

[28] J. Zhao and J. Wang. Adaptive observer for joint estimation of oxygen fractions and blend level in biodiesel fueled engines. IEEE Transactions on Control Systems Technology, 23(1):80-90, 2015.

[29] F. Castillo, E. Witrant, V. Talon, and L. Dugard. Simultaneous air fraction and low-pressure EGR mass flow rate estimation for diesel engines. In IFAC Proceedings Volumes (IFAC-PapersOnline), pages 731-736, 2013.

[30] Rudolph Emil Kalman. A new approach to linear filtering and prediction problems. Journal of basic Engineering, 82(1):35-45, 1960.

[31] Osvaldo Barbarisi, G Alessandro, and G Luigi. An extended Kalman observer for the in-cylinder air mass flow estimation. In Proceedings of MECA02 International Workshop on Diagnostics in Automotive Engines and Vehicles, Oct., Fisciano SA, pages 1-14, 2002.

[32] A. Dutka, H. Javaherian, and M. J. Grimble. State-dependent Kalman filters for robust engine control. In Proceedings of the American Control Conference, volume 2006, pages 1185-1190, 2006.

[33] T. Polóni, B. Rohaĺ-Ilkiv, and T. Arne Johansen. Mass flow estimation with model bias correction for a turbocharged diesel engine. Control Engineering Practice, 23(1):22-31, 2014.

[34] P. Kyrtatos, K. Hoyer, P. Obrecht, and K. Boulouchos. Apparent effects of in-cylinder pressure oscillations and cycle-to-cycle variability on heat release rate and soot concentration under long ignition delay conditions in diesel engines. International Journal of Engine Research, 15(3):325-337, 2014.

[35] P. Kyrtatos, C. Brückner, and K. Boulouchos. Cycle-to-cycle variations in diesel engines. Applied Energy, 171:120-132, 2016.

[36] J.B. Heywood. Internal Combustion engine fundamentals. McGraw-Hill, New York, 1988.

[37] C. Guardiola, B. Pla, P. Bares, and A. Barbier. An analysis of the incylinder pressure resonance excitation in internal combustion engines. Applied Energy, 228:1272-1279, 2018.

[38] C. S. Draper. The physical effects of detonation in a closed cylindrical chamber. Technical report, National Advisory Committee for Aeronautics, 1938.

[39] F. Payri, A. Broatch, B. Tormos, and V. Marant. New methodology for in-cylinder pressure analysis in direct injection diesel engines - application to combustion noise. Measurement Science and Technology, 16(2):540$547,2005$. 